\title{
The Solution to a Nonlinear Lamm Equation in the Faxén Approximation
}

\author{
Irwin H. Billick and George H. Weiss*
}

(October 5, 1965)

\begin{abstract}
An exact solution in the Faxén approximation is given for the Lamm equation in which the sedimentation coefficient is related to concentration as $s=s_{0}(1-k c)$. It is shown that the solution in this case can be expressed in terms of the solution to the linear case $(k=0)$ with a modified argument. The boundary sharpening phenomenon expresses itself very clearly in the solution presented here.
\end{abstract}

Key Words: Sedimentation, Lamm equation, concentration dependence, nonlinear.

Solutions of the Lamm equation for constant diffusion and sedimentation coefficients have found wide application in the analysis of data from the ultracentrifuge [1]. ${ }^{1}$ It is known that both the sedimentation and the diffusion coefficients depend on concentration, and in particular, the sedimentation coefficient can be approximated by

$$
s=s_{0} /(1+k c)
$$

where $s_{0}$ is the sedimentation coefficient at infinite dilution and $k$ is a constant which is a measure of nonlinearity. Fujita has shown that if the relation of eq (1) is replaced by

$$
s=s_{0}(1-k c)
$$

while the diffusion coefficient remains independent of concentration, the resulting Lamm equation can be linearized rigorously [2]. Fujita solved this Lamm equation in the Faxén regime by an approximation technique. It is the purpose of this paper to show that an exact solution of the Lamm equation is possible in the Faxén regime, and that the solution for the nonlinear case can be written entirely in terms of the solution to the linear problem. The technique exploited in this paper was originally suggested in a preliminary note [3] although the result given there contains an error. Our solution then enables one to derive an expansion in powers of the time variable to as high a degree as desired, whereas Fujita's original solution was limited in this respect. The Lamm equation can also be solved with the ansatz of eq (2) in the rectangular approximation [4], and an Archibald solution which takes account of boundary conditions can be written [5]. A detailed comparison between solutions of the Lamm equation with the two forms of sedimentation coefficient given by eqs (1) and (2) will be presented elsewhere [6].

\footnotetext{
*National Cancer Institute, National Institutes of Health, Bethesda, Md.

${ }^{1}$ Figures in brackets indicate the literature references at the end of this paper.
} 
The Lamm equation will be written

$$
\frac{\partial c}{\partial t}=\frac{1}{r} \frac{\partial}{\partial r}\left(r D \frac{\partial c}{\partial r}-s_{0} \omega^{2} r^{2}(1-k c) c\right)
$$

in terms of the solute concentration $c$, the diffusion coefficient $D$, sedimentation coefficient $s_{0}$, and angular frequency $\omega$. Let $r_{0}$ be the radial position of the meniscus, and $c_{0}$ be the initia' concentration. Then the transformations

$$
\theta=c / c_{0}, \quad \epsilon=2 D /\left(s_{0} \omega^{2} r_{0}^{2}\right) \quad x=\left(r / r_{0}\right)^{2}, \quad \tau=2 \omega^{2} s_{0} t \quad \alpha=k c_{0}
$$

convert eq (3) into

$$
\frac{\partial \theta}{\partial \tau}=\frac{\partial}{\partial x}\left\{\epsilon x \frac{\partial \theta}{\partial x}-x \theta(1-\alpha \theta)\right\}
$$

This equation can be linearized by introducing a new dependent variable $u(x, \tau)$ by

$$
\theta(x, \tau)=\frac{\epsilon}{\alpha} \frac{\partial}{\partial x} \ln u(x, \tau) .
$$

It proves convenient to also define new independent variables by

$$
z=2\left(x e^{-\tau}\right)^{1 / 2}, \quad \zeta=1-e^{-\tau}
$$

which, together with eqs (5) and (6) implies that $u$ satisfies

$$
\frac{1}{\epsilon} \frac{\partial u}{\partial \zeta}=\frac{\partial^{2} u}{\partial z^{2}}-\frac{1}{z} \frac{\partial u}{\partial z}
$$

which is to be solved with the initial condition

$$
u(\sigma, 0)=1, \quad 0 \leqslant \sigma \leqslant 2=\exp \frac{\alpha}{4 \epsilon}\left(\sigma^{2}-4\right), \quad \sigma>2 .
$$

We can solve eq (8) by a separation of variables. A general solution for which $\partial u / \partial z$ remains finite at $x=0$ is

$$
u(z, \zeta)=A+B z^{2}+z \int_{0}^{\infty} g(\lambda) J_{1}(\lambda z) e^{-\lambda^{2} \epsilon \zeta} d \lambda
$$

where $A$ and $B$ are constants, and $g(\lambda)$ is to be determined from initial conditions. Notice that the first two terms are solutions to the homogeneous equation obtained by setting $\partial u / \partial \zeta=0$. Initially we have

$$
u(z, 0)-A-B z^{2}=z \int_{0}^{\infty} g(\lambda) J_{1}(\lambda z) d \lambda
$$

where $g(\lambda)$ can be calculated by regarding the right-hand side as a Hankel transform [6]. In this way we find

$$
g(\lambda)=\lambda \int_{0}^{\infty} J_{1}(\lambda \sigma)\left[u(\sigma, 0)-A-B \sigma^{2}\right] d \sigma .
$$


Hence $u(z, \zeta)$ is given by [7]

$$
\begin{aligned}
& u\left(z_{1} \zeta\right)=A+B z^{2}+z \int_{0}^{\infty}[\left.u(\sigma, 0)-A-B \sigma^{2}\right] d \sigma \int_{0}^{\infty} \lambda J_{1}(\lambda \sigma) J_{1}(\lambda z) e^{-\lambda^{2} \epsilon \zeta} d \lambda \\
&=A+B z^{2}+\frac{z}{2 \epsilon \zeta} e^{-\frac{z^{2}}{4 \epsilon \zeta}} \int_{0}^{\infty} e^{-\frac{\sigma^{2}}{4 \epsilon \zeta}} \quad I_{1}\left(\frac{z \sigma}{2 \epsilon \zeta}\right)\left[u(\sigma, 0)-A-B \sigma^{2}\right] d \sigma
\end{aligned}
$$

where $I_{1}(\mu)$ is a Bessel function of the first kind, with imaginary argument.

Since

$$
\int_{0}^{\infty} \sigma^{2} e^{-\frac{\sigma^{2}}{4 \epsilon \zeta}} I_{1}\left(\frac{z \sigma}{2 \epsilon \zeta}\right) d \sigma=2 z \epsilon \zeta e^{\frac{z^{2}}{4 \epsilon \zeta}}
$$

we see that the terms containing $B$ cancel. The constant $A$ can be determined by requiring that the limiting solution for $\alpha \rightarrow 0$ agree with the known Faxén solution for constant $s$ and $D$. It can easily be verified that this is equivalent to setting $A=1$. Hence an expression for $u(z, \zeta)$ is

$$
u(z, \zeta)=1+\frac{z}{2 \epsilon \zeta} \int_{2}^{\infty} e^{-\frac{\left(z^{2}+\sigma^{2}\right)}{4 \epsilon \zeta}} I_{1}\left(\frac{z \sigma}{2 \epsilon \zeta}\right)\left[e^{\frac{\alpha}{4 \epsilon}\left(\sigma^{2}-4\right)}-1\right] d \sigma .
$$

It will prove convenient for later purposes to perform an integration by parts, noting that $I_{1}(y)$ $=d I_{0}(y) / d y$. This leads to the expression

$$
u(z, \zeta)=1+\frac{1}{2 \epsilon \zeta} \int_{2}^{\infty} \sigma e^{-\frac{\left(z^{2}+\sigma^{2}\right)}{4 \epsilon \zeta}} I_{0}\left(\frac{z \sigma}{2 \epsilon \zeta}\right)\left[(1-\alpha \zeta) e^{\left.\frac{\alpha}{4 \epsilon}\left(\sigma^{2}-4\right)-1\right] d \sigma}\right.
$$

Differentiation according to eq (6) together with an integration by parts leads to an expressione for $\theta(z, \zeta)$,

$$
\theta(z, \zeta)=\frac{1-\zeta}{2 \epsilon \zeta u(z, \zeta)} \int_{2}^{\infty} \sigma e^{-\frac{\left(z^{2}+\sigma^{2}\right)}{4 \epsilon \zeta}} I_{0}\left(\frac{z \sigma}{2 \epsilon \zeta}\right) e^{\frac{\alpha}{4 \epsilon}\left(\sigma^{2}-4\right)} d \sigma
$$

When $\alpha=0$ this relation reduces to the known Faxén solution, which will be denoted by $\theta_{0}(z, \zeta)$ :

$$
\theta_{0}(z, \zeta)=\frac{1-\zeta}{2 \epsilon \zeta} \int_{2}^{\infty} \sigma e^{-\frac{\left(\sigma^{2}+z^{2}\right)}{4 \epsilon \zeta} I_{0}}\left(\frac{z \sigma}{2 \epsilon \zeta}\right) d \sigma
$$

We now notice that all of the integrals appearing in the expression for $\theta(z, \zeta)$ have the same general form of an exponential of a quadratic in $\sigma$, multiplied by the common factor

$$
\sigma \exp \left[-\frac{\left(\sigma^{2}+z^{2}\right)}{4 \epsilon \zeta}\right] I_{0}\left(\frac{z \sigma}{2 \epsilon \zeta}\right)
$$

Hence all of the integrals are expressible in terms of $\theta_{0}$ provided that the arguments are properly chosen. After some algebra it is found that

$$
\theta(z, \zeta)=\frac{1-\zeta}{1-(\alpha+1) \zeta} \frac{e^{-\frac{\alpha}{\epsilon}\left[1-\frac{z^{2}}{4(1-\alpha \zeta)}\right] \theta_{0}\left(z^{*}, \zeta^{*}\right)}}{1+\frac{1-\alpha \zeta}{1-(\alpha+1) \zeta} e^{-\frac{\alpha}{\epsilon}\left[1-\frac{z^{2}}{4(1-\alpha \zeta)}\right]} \theta_{0}\left(z^{*}, \zeta^{*}\right)-\frac{1}{1-\zeta} \theta_{0}(z, \zeta)}
$$


where

$$
z^{*}=\frac{z}{1-\alpha \zeta}, \zeta^{*}=\frac{\zeta}{1-\alpha \zeta}
$$

Simple properties of the solution follow immediately from the representation of eq (19). The parameter $\alpha / \epsilon$ which appears in the expression of eq (19) is commonly greater than 10 for values of $D, s$, and $\omega^{2}$ (which validate the approximation of eq (2)) and the Faxén approximation. Hence the exponential terms in eq (19) predominate when

$$
\frac{z^{2}}{4(1-\alpha \zeta)}-1
$$

is positive by an order of magnitude greater than $\epsilon / \alpha$. When this is the case we have

$$
\theta(z, \zeta) \sim \frac{1-\zeta}{1-\alpha \zeta}=\left[\alpha+(1-\alpha) e^{\tau}\right]^{-1}
$$

which defines the behavior of the plateau region. This can also be verified directly from eq (5). When the quantity of eq (2l) is negative by an order of magnitude greater than $\epsilon / \alpha, \theta(z, \zeta)$ is effectively equal to zero. The transition region occurs from values of $r$ for which

$$
\frac{z^{2}}{4(1-\alpha \zeta)}-1=0\left(\frac{\epsilon}{\alpha}\right)
$$

Thus, in the limit $\epsilon=0$ the transition region (from essentially zero to a plateau value) is very sharp, and for fixed $\epsilon$ and increasing $\alpha$ the transition region also becomes sharper, as indeed is well known. If the gradients are denoted by $G(z, \zeta)$ and $G_{0}(z, \zeta)$, i.e.,

$$
G(z, \zeta)=\frac{\partial \theta}{\partial\left(r / r_{0}\right)}, \quad G_{0}(z, \zeta)=\frac{\partial \theta_{0}}{\partial\left(r / r_{0}\right)}
$$

then we can write for $G(z, \zeta)$ :

$$
\begin{aligned}
G(z, \zeta)=\frac{1}{1-\alpha \zeta} \frac{G_{0}(z, \zeta)}{F\left(z, z^{*}, \zeta, \zeta^{*}\right)}+\frac{1-\zeta}{1-\alpha \zeta} \frac{\left(1-\frac{1}{1-\zeta} \theta_{0}(z, \zeta)\right)}{F^{2}\left(z, z^{*}, \zeta, \zeta^{*}\right)} \\
\quad \times\left\{\frac{\alpha}{4 \epsilon} \frac{(1-\zeta)^{1 / 2}}{1-(\alpha+1) \zeta} e^{-\frac{\alpha}{\epsilon}\left[1-\frac{z^{2}}{4(1-\alpha \zeta)}\right]} \theta_{0}\left(z^{*}, \zeta^{*}\right)+\frac{e^{-\frac{\alpha}{\epsilon}\left[1-\frac{z^{2}}{4(1-\alpha \zeta)}\right]}}{1-(\alpha+1) \zeta} G_{0}\left(z^{*}, \zeta^{*}\right)-\frac{1}{1-\zeta} G_{0}(z, \zeta)\right\}
\end{aligned}
$$

where

$$
F\left(z, z^{*}, \zeta, \zeta^{*}\right)=1+\frac{1-\alpha \zeta}{1-(\alpha+1) \zeta} e^{-\frac{\alpha}{\epsilon}\left[1-\frac{z^{2}}{4(1-\alpha \zeta)}\right]} \theta_{0}\left(z^{*}, \zeta^{*}\right)-\frac{1}{1-\zeta} \theta_{0}(z, \zeta)
$$

It is possible now to derive successive approximations to $\theta(z, \zeta)$ by expanding it in powers of $(\epsilon \zeta)^{1 / 2}$. That this is the natural parameter for the expansion will be seen later. Since $\theta(z, \zeta)$ depends only on $\theta_{0}(z, \zeta)$ we must first consider the expansion of the latter function for small $\zeta$. For small $\zeta$ the Bessel function $I_{0}\left(\frac{z \sigma}{2 \epsilon \zeta}\right)$ has the asymptotic expansion [7] 


$$
I_{0}\left(\frac{z \sigma}{2 \epsilon \zeta}\right) \sim \sqrt{\frac{\epsilon \zeta}{\pi z \sigma}} e^{\frac{z \sigma}{2 \epsilon \zeta}} \sum_{j=0}^{\infty} B_{j}\left(\frac{2 \epsilon \zeta}{z \sigma}\right)^{j}
$$

where

$$
B_{j}=\frac{(-1)^{j}}{2^{j} j !} \frac{\Gamma\left(j+\frac{1}{2}\right)}{\Gamma\left(-j+\frac{1}{2}\right)} .
$$

Therefore $\theta_{0}(z, \zeta)$ has the expansion

$$
\begin{aligned}
\theta_{0}(z, \zeta) \sim \frac{e^{-\tau}}{2 \sqrt{\pi z \epsilon \zeta}} \sum_{j=0}^{\infty} B_{j}\left(\frac{2 \epsilon \zeta}{z}\right)^{j} \int_{2}^{\infty} e^{-\frac{(\sigma-2)}{4 \epsilon \zeta}} & \frac{d \sigma}{\sigma^{j-1 / 2}} \\
& =\frac{e^{-\tau}}{\sqrt{2 \pi z}} \sum_{j=0}^{\infty} B_{j}\left(\frac{2 \epsilon \zeta}{z}\right)^{j} \int_{\frac{2-z}{\sqrt{2 \epsilon \zeta}}}^{\infty} e^{-u^{2} / 2} \frac{d u}{(z+u \sqrt{2 \epsilon \zeta})^{j-1 / 2}} .
\end{aligned}
$$

We now consider the integrals which appear in this series and expand them in powers of $(\epsilon \zeta)^{1 / 2}$. To carry out this expansion we write, for fixed $u$ and small $\zeta$

$$
(z+u \sqrt{2 \epsilon \zeta})^{-j+1 / 2}=\sum_{r=0}^{\infty}\left(\begin{array}{c}
-j+1 / 2 \\
r
\end{array}\right) u^{r}(2 \epsilon \zeta)^{r / 2} z^{-j-r+1 / 2} .
$$

This can be further transformed by noting the identity

$$
\left(\begin{array}{c}
-j+1 / 2 \\
r
\end{array}\right)=(-1)^{r}\left(\begin{array}{c}
r+j-3 / 2 \\
r
\end{array}\right) .
$$

Finally, combining eqs (29), (30), and (31) we find that the complete asymptotic expansion of the Faxén solution can be written as

$$
\theta_{0}(z, \zeta) \sim \frac{1-\zeta}{\sqrt{2 \pi}} \sum_{j=0}^{\infty} \sum_{m=2 j}^{\infty}(-1)^{m} B_{j}\left(\begin{array}{c}
m-j-3 / 2 \\
j-3 / 2
\end{array}\right) \frac{(2 \epsilon \zeta)^{m / 2}}{z^{m}} U_{m-2 j}\left(\frac{2-z}{\sqrt{2 \epsilon \zeta}}\right)
$$

where the $U_{r}(y)$ are defined by

$$
U_{r}(y)=\int_{y}^{x} u^{r} e^{-u^{2} / 2} d u .
$$

In particular, the first two terms of eq (32) yield the usual Faxén approximation

$$
\theta_{0} \sim \frac{e^{-\tau}}{\sqrt{2 \pi}}\left\{U_{0}\left(\frac{2-z}{\sqrt{2 \epsilon \zeta}}\right)+\frac{1}{z}\left(\frac{\epsilon \zeta}{2}\right)^{1 / 2} e^{-\frac{(2-z)^{2}}{4 \epsilon \zeta}}\right\}
$$

in which $U_{0}(y)$ can be expressed in terms of an error function. A complete asymptotic expansion for $\theta(z, \zeta)$ can now be obtained by combining eqs (19) and (32). Similarly, $\partial \theta / \partial\left(r / r_{0}\right)$ is expressible in terms of $\theta_{0}(z, \zeta)$ and its derivative, $G_{0}(z, \zeta)$, which has the expansion

$$
\begin{aligned}
G_{0}(z, \zeta) \sim \frac{e^{-\tau}}{\sqrt{2 \pi}} \sum_{j=0}^{\infty} \sum_{m=2 j}^{\infty}(-1)^{m} B_{j}\left(\begin{array}{c}
m-j-3 / 2 \\
j-3 / 2
\end{array}\right) & \frac{(2 \epsilon \zeta)^{m / 2}}{z^{m}} \\
& \times\left\{-\frac{m}{z} U_{m-2 j}\left(\frac{2-z}{\sqrt{2 \epsilon \zeta}}\right)+\frac{(2-z)^{m-2 j}}{(2 \epsilon \zeta)^{\frac{m-2 j+1}{2}}} e^{-\frac{(2-z)^{2}}{4 \epsilon \zeta}}\right\} .
\end{aligned}
$$

The lowest order Faxén approximation to $\theta(z, \zeta)$ is

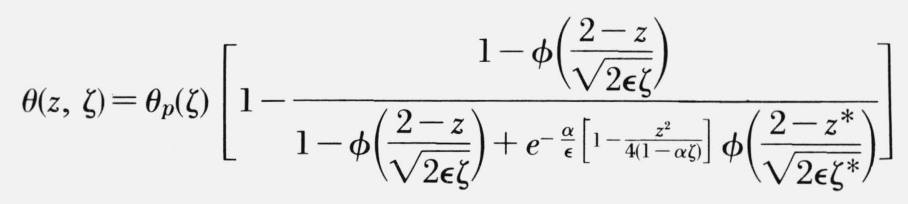


where $\theta_{p}(\zeta)$ is the normalized plateau concentration (eq $\left.(22)\right)$ and $\phi(\mu)=(2 \pi)^{-1 / 2} \int_{\mu}^{\infty} \exp \left(-x^{2} / 2\right)$ $d x$ is the complementary error function. To this approximation, the gradient is

$$
\begin{aligned}
\frac{\partial \theta}{\partial\left(r / r_{0}\right)}=2 e^{-\tau / 2} \frac{\partial \theta}{\partial z}= & \frac{e^{-\tau / 2-\frac{\alpha}{\epsilon}\left[1-\frac{z^{2}}{4(1-\alpha \zeta)}\right]}}{D} \\
& \times\left\{\frac{1}{\sqrt{\pi \epsilon \zeta}} \phi\left(\frac{2-z^{*}}{\sqrt{2 \epsilon \zeta^{*}}}\right) e^{-\frac{\left(z-z^{*}\right)^{2}}{4 \epsilon \zeta^{*}}}+\frac{1}{\sqrt{\pi \epsilon \zeta^{*}}}\left(1-\phi\left(\frac{2-z}{\sqrt{2 \epsilon \zeta}}\right)\right) e^{-\frac{(2-z)^{2}}{4 \epsilon \zeta}}\right. \\
& \left.\quad+\frac{\alpha z}{\epsilon(1-\alpha \zeta)} \phi\left(\frac{2-z^{*}}{\sqrt{2 \epsilon \zeta^{*}}}\right)\left(1-\phi\left(\frac{2-z}{\sqrt{2 \epsilon \zeta}}\right)\right)\right\}
\end{aligned}
$$

where

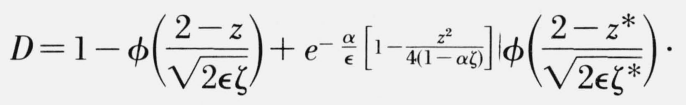

When $\alpha$ is set equal to 0 eq (37) reduces to the known Faxén solution.

Fujita [2] showed that in his approximation the position of the maximum is given by

$$
r_{\max }=r_{0} e^{\tau}\left[1-\alpha\left(1-e^{-\tau}\right)\right]
$$

The result of eq (19) implies that this is modified to

$$
r_{\max }=r_{0} e^{\tau}\left[1-\alpha\left(1-e^{-\tau}\right)\right][1+f] .
$$

The factor $f$ is generally of the order of 0.1 or less and therefore Fujita's result for the position of the maximum gradient is sufficient for all practical purposes. It also follows that Fujita's calculations for the height-area ratio are sufficiently good for practical purposes.

\section{References}

[1] H. Fujita, Mathematical Theory of Sedimentation Analysis (Academic Press, New York, 1962).

[2] H. Fujita, Effects of a concentration dependence of the sedimentation coefficient in velocity ultracentrifugation, J. Chem. Phys. 24, 1084 (1956).

[3] I. H. Billick, G. H. Weiss, Exact Faxén solution for centrifugation when sedimentation depends linearly on concentration, Nature, 201, 912 (1964).

[4] G. H. Weiss, D. A. Yphantis, Rectangular approximation for concentration dependence in the ultracentrifuge, J. Chem. Phys. 42, 2117 (1965).

[5] G. H. Weiss, An Archibald type solution to a non-linear Lamm equation, Nature 202, 792 (1964).

[6] M. Dishon, G. H. Weiss, and D. A. Yphantis, Numerical solutions to the Lamm equation, to appear in Biopolymers.

[7] I. M. Ryshik, I. S. Gradstein, Tables of Series, Products, and Integrals, 2d edition (Plenum Press, New York, 1963).

(Paper 70Al-383) 